\title{
The facilitative effects of glucose ingestion on memory retrieval in younger and older adults: is task difficulty or task domain critical?
}

\author{
Leigh M. Riby ${ }^{1}$, Hazel McMurtrie ${ }^{1}$, Jonathan Smallwood ${ }^{2}$, Carrie Ballantyne ${ }^{1}$, Andrew Meikle ${ }^{1}$ and \\ Emily Smith ${ }^{1}$ \\ ${ }^{1}$ Department of Psychology, Glasgow Caledonian University, 70 Cowcaddens Road, Glasgow G4 OBA, UK \\ ${ }^{2}$ Department of Psychology, University of British Columbia, 2329 West Mall, Vancouver V6T 1Z4, Canada
}

(Received 23 May 2005 - Revised 17 October 2005 - Accepted 18 October 2005)

\begin{abstract}
The ingestion of a glucose-containing drink has been shown to improve cognitive performance, particularly memory functioning. However, it remains unclear as to the extent to which task domain and task difficulty moderate the glucose enhancement effect. The aim of this research was to determine whether boosts in performance are restricted to particular classes of memory (episodic $v$. semantic) or to tasks of considerable cognitive load. A repeated measures ( $25 \mathrm{~g}$ glucose $v$. saccharin), counterbalanced, double-blind design was used with younger and older adults. Participants performed a battery of episodic (e.g. paired associate learning) and semantic memory (e.g. category verification) tasks under low and high cognitive load. Electrophysiological measures (heart rate and galvanic skin response) of arousal and mental effort were also gathered. The results indicated that whilst glucose appeared to aid episodic remembering, cognitive load did not exaggerate the facilitative effect. For semantic memory, there was little evidence to suggest that glucose can boost semantic memory retrieval even when the load was manipulated. One exception was that glucose facilitated performance during the difficult category fluency task. Regardless, the present findings are consistent with the domain-specific account in which glucose acts primarily on the hippocampal region, which is known to support episodic memory. The possible contribution of the hippocampus in semantic memory processing is also discussed.
\end{abstract}

Glucose: Episodic memory: Semantic memory: Cognitive performance: Task difficulty

Recently there has been a growth in research examining how nutritional factors can contribute to boosts in cognitive functioning. For example, the intake of everyday substances such as glucose (see Messier, 2004 for review; Riby, 2004 for meta-analysis), dietary carbohydrates (e.g. Kaplan et al. 2000) and herbal extracts (e.g. Kennedy \& Scholey, 2003) have been found to facilitate performance on a variety of cognitive tasks. The impact of a glucose-containing drink on performance has in particular received a great deal of attention. Indeed, research has indicated improvements on cognitive tasks in a variety of populations such as younger adults (e.g. Foster et al. 1998), older adults (e.g. Hall et al. 1989), Alzheimer's disease (Manning et al. 1993), Down syndrome (Manning et al. 1998) and schizophrenia (Fucetola et al. 1999). Whilst this literature has supported the idea that man can benefit from a moderate increase in blood glucose, it has proven difficult to reach firm conclusions from the literature because of the diversity of methods used. So, although there appears to be great promise for the use of glucose as a cognitive enhancer, there is debate as to whether glucose especially benefits memory functioning, particularly episodic remembering. In addition, it is as yet unclear whether enhancement is closely related to the difficulty of the tasks being performed (Scholey et al. 2001). To address these issues, we investigated whether the type of memory task (episodic $v$. semantic memory) or the difficulty of the task (easy $v$. hard tasks) is the most important moderator of the glucose facilitation effect in both younger and older adults.

An illustration of the selectivity of memory enhancement by glucose was provided by Foster et al. (1998). In their study, young healthy participants performed a battery of cognitive tasks after the consumption of either a $25 \mathrm{~g}$ glucose drink or a control solution (i.e. saccharin). The test battery consisted of several different memory tasks, including immediate, short-delay and long-delay long-term memory (free recall, cued recall and recognition), the Rey-Osterrich complex figure drawing task (long-term memory for nonverbal materials) and digit span (working memory). Overall, there was a significant glucose facilitation effect on performance of long-term memory free and cued recall tasks. No other significant improvements in memory performance were observed. Thus, the enhancing effects of glucose appear to be selective in that marked enhancement is restricted to tests of long-term verbal episodic memory. However, studies have indicated that both a memory component and sufficient task demands are necessary to observe facilitation (see Messier, 2004 for review). In a similar vein, Hall et al. (1989) compared younger and older adults on a 
battery of cognitive tests. Consistent with previous research significant boosts in performance were observed on episodic remembering. Importantly, the effect was more pronounced for older adults. Indeed, the specificity of the glucose effect to episodic memory and the finding of greater enhancement in an elderly population are consistent with our physiological knowledge of memory. In particular, the cholinergic system has an important role to play and may explain memory problems in ageing (Dunnett, 1991). Messier et al. (1997) argued strongly that glucose could facilitate hippocampal function and more specifically cholinergic activity by increasing the synthesis of acetylcholine. Recently, however, Scholey et al. (2001) argued that it is unlikely that the cholinergic system is solely involved in the glucose facilitation effect. Moreover, researchers have suggested interactions with other neurotransmitter substances, which points to a more global effect of glucose (e.g. dopamine; Saller \& Kreamer, 1991). In a recent meta-analysis Riby (2004) also found glucose facilitated performance on semantic memory tasks. In that study, a large overall effect size $(d 0.73)$ was observed, which provided evidence for boosts in declarative memory in general. It should be noted that although Riby (2004) identified facilitation on semantic memory tasks the sample was low (four studies included in the analysis) and previous research has tended to use verbal fluency as a measure of semantic memory, which arguably could be described as a test of frontal lobe function. Here we extend previous research by employing several measures of semantic memory processing.

Task difficulty has been proposed as a significant factor influencing the magnitude of glucose-induced enhancement rather than task domain. For example, Kennedy \& Scholey (2000) found a relationship between subjective measures of task difficulty and the magnitude of glucose enhancement. In that study, young healthy participants performed two serial subtraction tasks (Serial Threes and Serial Sevens working memory tasks) and a Word Retrieval task. In the task perceived as most difficult by the participant (i.e. Serial Sevens) greater enhancement of glucose was evident. Kennedy \& Scholey (2000) concluded that their findings provided further evidence that glucose selectively enhances tasks of a more demanding nature. Importantly, their study employed a physiological measure of task demand, namely heart rate. Overall, more cognitively demanding tasks increased heart rate. Furthermore, Scholey et al. (2001) demonstrated a marked reduction in blood glucose while performing cognitive demanding tasks. Arguably, the ingestion of a glucose-containing drink could serve to maintain performance under such demanding conditions. So, the aforementioned finding of greater enhancement on episodic memory tasks might be consistent with a cognitive demand account, rather than task domain. That is, episodic remembering may generally require more effortful processing (i.e. the retrieval of detailed item and contextual information) for task competition and benefit more from the intake of a glucose-containing drink. Furthermore, the finding of greater facilitation in an elderly population is consistent with the cognitive demand hypothesis. Presumably, in such populations cognitive tasks are generally more difficult, due to a reduction in cognitive resources (e.g. Craik, 1986), and therefore there is more scope for glucoserelated facilitation.
In summary, previous research on the effects of task domain (in the present study episodic $v$. semantic memory) is inconclusive because there are no studies that have investigated both task types while the difficulty is manipulated within the same experiment. In addition, compared with episodic memory the impact of glucose on semantic memory processing has been neglected. The aims of the present experiment were to investigate further whether glucose enhancement effects are restricted to episodic memory and the impact of task difficulty in both younger and older adults. Different measures of task demand were employed since previous research has indicated that the type of difficulty manipulation may be crucial (e.g. Meikle et al. 2005). So, there may be some limits on the forms of difficulty that are susceptible to enhancement. The age manipulation in the current investigation reflects: 1) the well-established findings in the ageing literature that older human subjects (and rats) perform at a poorer standard on tasks of memory; 2) that the enhancing effects of glucose may be greater in a 'deficit' population (Messier \& Gagnon, 1996). Kennedy \& Scholey (2000) effectively employed a physiological measure of task demand (heart rate). Thus, we use a similar strategy and use two physiological measures (heart rate and galvanic skin response (GSR)) as a further assessment of the impact of task difficulty.

\section{Method \\ Participants}

A total of thirteen older adults (mean age 68 (SD 5.9) years) and fourteen younger adults (mean age 30.1 (SD 4.6) years) participated in the experiment. On average the younger adults were better educated (17.3 v. 13.1 years of education, respectively, $t(25) 3 \cdot 8, P<0 \cdot 01)$. There was no difference between scores on the National Adult Reading Test (raw scores of 39.1 and 36.3 for younger and older adults, respectively, $P>0.05)$. Older adults were screened for dementia using the Mini-Mental State Exam (Folstein et al. 1975). The present study was approved by the Department of Psychology ethics committee. All participants gave informed consent to take part in the experiment.

\section{Design and treatment}

Each participant acted as his or her own control in a repeated measures design, receiving one of two possible treatments ( $25 \mathrm{~g}$ glucose or $38 \mathrm{mg}$ saccharin solution) in a counterbalanced order. Both the experimenter and participant were blind to the treatment drink. To ensure that all drinks had a similar 'mouthfeel' and to control for sweetness reinforcement effects, the treatment drinks contained sugar-free whole orange squash (glucose $30 \mathrm{ml}$; saccharin $45 \mathrm{ml}$ ) dissolved in $250 \mathrm{ml}$ water. The choice of these solutions was based on previous research where similar matching of drinks has been employed (e.g. Meikle et al. 2005). Participants were instructed not to eat and to drink only water from midnight the previous night to ensure their blood glucose was at fasting levels. All testing was carried out between 09.00 and 13.00 hours. 


\section{Materials}

Episodic memory tasks. A master list was constructed from concrete (imagery scores greater than 500) and abstract (imagery score below 350) words, between four and seven letters in length, selected from the MRC Psycholinguistic Database (Coltheart, 1981). The first episodic memory task consisted of four lists of twelve unrelated concrete paired associates (e.g. tree-car), selected at random from the master list. Two of the word lists were presented under single and two under dual task encoding conditions. In the dual task encoding condition a pack of Wisconsin Card Sorting Cards was used as stimuli. There were two versions of the stimuli that were used in a counterbalanced order across glucose and saccharin conditions, with the imagery rating and word frequency taken into consideration for each list (version 1 means: imagery rating 519, word frequency 130 , word length $5 \cdot 5$; version 2 means: imagery rating 509, word frequency 119 , word length 5.3). A second episodic memory task consisted of two lists, each of twelve concrete words and twelve abstract words, each selected at random from the master list. Again, there were two versions of the stimuli, the presentation of which were counterbalanced across conditions, with the imagery rating and word frequency taken into consideration for each list (version 1 abstract word means: imagery rating 314 , word frequency 156 , mean word length $5 \cdot 1$; version 2 abstract word means: imagery rating 305, word frequency 186 , word length $5 \cdot 2$; version 1 concrete word means: imagery rating 491, word frequency 144 , word length $5 \cdot 3$; version 2 concrete word means: imagery rating 528 , mean word frequency 127 , word length $5 \cdot 3$ ).

Computerized semantic verification task. The categories and category members were selected from the Belfast Category Norms (Brown, 1978). A master list of 120 category names and an exemplar pair list were generated, which included half typical (e.g. animal-dog) and half atypical (e.g. animal-walrus) category-exemplar pairs. In addition, six category-exemplar pairs were used for the practice session.

\section{Procedure}

Each participant attended two sessions (1 week apart), each lasting for approximately $1 \mathrm{~h}$. Table 1 illustrates the sequence of events. After fasting overnight, participants received either

Table 1. Testing schedule

\begin{tabular}{ll}
\hline Time (min) & Task \\
\hline 0 & Drink administration \\
& Instructions \\
& Set-up of electrophysiological equipment \\
& Paired associate learning and immediate cued \\
& recall under single and dual task conditions \\
& Learning of abstract and concrete words \\
& followed by immediate free recall \\
& Verbal fluency \\
& Category fluency \\
& Computerized semantic verification task \\
& Delayed cued recall of paired associates \\
& Delayed free recall of abstract and concrete words \\
& Session ends
\end{tabular}

For details of subjects and procedures, see p. 415 the glucose or saccharin drink. A fasting compliance questionnaire was administered before each testing session. Testing commenced $10 \mathrm{~min}$ after ingestion. During this $10 \mathrm{~min}$ period electrophysiological equipment was attached to the participant to measure arousal throughout the testing session. This comprised three electrodes for the measurement of electrocardiograms and two electrodes placed on the index and middle fingers of the left hand to measure GSR. Electrocardiograms and GSR data were collected continuously throughout the experimental session using instrumentation supplied by Biopac Systems, Inc. Goleta, USA.

Episodic memory task 1 - paired associate learning under single (easy) and dual (difficult) task conditions. Prior to the experimental task, participants received detailed instructions with regard to the nature of the episodic task. In each session there were four study phases. Paired associates were presented verbally at a rate of one pair per $6 \mathrm{~s}$. Immediately after each study phase, participants undertook cued recall tests in which they were given the first word of each word pair and were required to recall the appropriate word to complete the pair. On completion of all subsequent tasks (described later) the participants' delayed cued recall was tested using the same method. In the dual task condition, participants were required to sort cards into different coloured piles whilst still listening to, and attempting to memorize, the word lists. Participants were instructed that the card sorting and learning of the paired associates were equally important. There were a total of 128 cards depicting figures of varying forms (crosses, circles, triangles or stars), colours (red, blue, yellow or green) and numbers (one, two, three or four).

Episodic memory task 2 - memory for concrete (easy) and abstract (difficult) words. Prior to the experimental task, participants received detailed instructions with regard to the nature of the episodic task. In each session there were four study blocks. Single words were presented verbally at a rate of one per $3 \mathrm{~s}$. Immediately after each study phase, participants were asked to free recall as many of the original words as they could remember. On completion of all other subsequent tasks (described later) participants' delayed free recall was tested by using the same method.

Semantic memory task 1 - word and category fluency. There were two versions of each task, which were administered in a counterbalanced order across sessions. Version one of the word fluency tasks required the participants to name as many words as they could recall which began with the letters $\mathrm{C}$ (easy) followed by $\mathrm{L}$ (difficult) within a $1 \mathrm{~min}$ period for each letter. In version two, participants were asked to name words beginning with $\mathrm{P}$ (easy) followed by $\mathrm{W}$ (difficult). The two versions were matched based on the Multilingual Aphasia Examination (see Lezak, 1995). Version one of the Category Fluency task required the participants to name as many vegetables (easy) and politicians (difficult) as possible within a $1 \mathrm{~min}$ period for each category. In version two, participants were asked to name colours (easy) and types of dance (difficult). The two versions were matched using the Belfast Category Norms (Brown, 1978).

Semantic memory task 2 - computerized semantic verification task. Prior to the experimental session each participant received a practice session of one block. The practice session consisted of six category-exemplar pairs presented for $5 \mathrm{~s}$ with an inter-stimulus interval of $500 \mathrm{~ms}$. Three of the pairs 
required a 'true' response (e.g. animal-horse) and three of the pairs a false response (e.g. fruit-tiger). There were four experimental blocks each containing thirty category-exemplar pairs; half requiring true responses and half false responses. Responses were made on a computer keyboard.

\section{Results}

A series of ANOVA were carried out on the performance scores summarized in Table 2. Time of day was initially included in the analyses but this factor had no impact on the results so is not discussed further.

\section{Episodic memory}

Memory performance for immediate cued recall under both single and dual task conditions were analysed in a 2 (young $v$. old $) \times 2$ (glucose $v$. saccharin $) \times 2$ (single $v$. dual $)$ ANOVA. This analysis revealed greater recall in the glucose compared with saccharin condition $(F(1,25)$ 4.61, mean square error (MSE) 6.20, $P<0.05$ ), greater recall in the single compared with dual task condition $(F(1,25) 46 \cdot 35$, MSE 10.49, $P<0.01)$ and greater recall for younger compared with older adults $(F(1,25) 32 \cdot 59$, MSE $83 \cdot 17, P<0 \cdot 01)$. The interaction between difficulty (single $v$. dual) and treatment demonstrated that glucose facilitation was the largest in the single task condition $(F(1,25) 4.65$, MSE 2.94, $P<0.05)$. All other interactions were unreliable. A separate ANOVA was conducted on the card-sorting data across treatment and age group. No main effects or interactions were significant.

Memory performance for delayed cued recall under both single and dual task conditions was analysed in a 2 (young $v$. old $\times 2$ (glucose $v$. saccharin $) \times 2$ (single $v$. dual $)$ ANOVA. This analysis revealed greater recall under single task conditions $(F(1,25) 24 \cdot 4$, MSE 7.73, $P<0.01)$ and greater recall for younger compared with older adults $(F(1,25) 36 \cdot 0$, MSE 73.77, $P<0.01)$. The interaction between treatment and age group approached significance $(F(1,25) 3 \cdot 40$, MSE $6 \cdot 25, P=0 \cdot 07)$. This interaction demonstrated a trend toward greater recall in the glucose condition for younger adults only. All other main effects and interactions were unreliable.

Memory performance for immediate free recall for concrete (easy) and abstract (hard) words was analysed in a 2 (young $v$. old $) \times 2$ (glucose $v$. saccharin) $\times 2$ (concrete $v$. abstract $)$ ANOVA. This analysis revealed greater recall for concrete compared with abstract words $(F(1,25)$ 48.7, MSE 3.73, $P<0.01)$ and greater recall for younger compared with older adults $(F(1,25) 19 \cdot 85$, MSE $34 \cdot 47, P<0 \cdot 01)$. The interaction between difficulty (concrete $v$. abstract) and age group demonstrated a greater fall between concrete and abstract words for younger adults $(F(1,25) 14 \cdot 24$, MSE $3 \cdot 73, P<0 \cdot 01)$. Importantly, there was a significant three-way interaction between treatment, difficulty and age group. Analyses of simple interaction effects using the Bonferroni procedure revealed a significant two-way interaction between treatment and difficulty for older adults $(P<0 \cdot 05)$. This interaction demonstrated that glucose facilitated older adults' free recall rates for concrete words only.

Memory performance for delayed free recall for concrete (easy) and abstract (hard) words was analysed in a 2 (young $v$. old $) \times 2$ (glucose $v$. saccharin) $\times 2$ (concrete $v$. abstract $)$ ANOVA. This analysis revealed greater recall for concrete compared with abstract words $(F(1,25) 34 \cdot 4$, MSE $7 \cdot 16$, $P<0.01)$ and greater recall for younger compared with older adults $(F(1,25) 25 \cdot 60$, MSE $36 \cdot 13, P<0 \cdot 01)$. The interaction between difficulty (concrete $v$. abstract) and age group demonstrated a greater fall in recall rates in the difficult condition for younger adults $(F(1,25) 7 \cdot 45$, MSE $7 \cdot 16, P<0 \cdot 05)$. In line

Table 2. Performance for episodic and semantic memory across age group and treatment $†$ (Mean values and standard deviations)

\begin{tabular}{|c|c|c|c|c|c|c|c|c|}
\hline & \multicolumn{4}{|c|}{ Younger adults ( $n$ 14) } & \multicolumn{4}{|c|}{ Older adults ( $n$ 13) } \\
\hline & \multicolumn{2}{|c|}{ Saccharin } & \multicolumn{2}{|c|}{ Glucose } & \multicolumn{2}{|c|}{ Saccharin } & \multicolumn{2}{|c|}{ Glucose } \\
\hline & Mean & SD & Mean & SD & Mean & SD & Mean & SD \\
\hline \multicolumn{9}{|l|}{ Episodic memory } \\
\hline Immediate cued recall - single & $17 \cdot 6$ & $4 \cdot 8$ & $20 \cdot 3^{*}$ & 3.4 & $8 \cdot 7$ & $5 \cdot 3$ & $9 \cdot 5^{\star}$ & $4 \cdot 8$ \\
\hline Immediate cued recall - dual & $14 \cdot 5$ & $6 \cdot 2$ & $15 \cdot 2$ & $5 \cdot 6$ & $4 \cdot 7$ & 4.9 & $4 \cdot 6$ & $5 \cdot 1$ \\
\hline Immediate free recall - concrete & $18 \cdot 7$ & 3.5 & $18 \cdot 8$ & $3 \cdot 9$ & $11 \cdot 5$ & $2 \cdot 9$ & $13 \cdot 1^{*}$ & $3 \cdot 3$ \\
\hline Immediate free recall - abstract & 14.4 & $2 \cdot 9$ & $15 \cdot 1$ & $4 \cdot 2$ & $11 \cdot 3$ & 3.8 & $10 \cdot 9$ & $2 \cdot 4$ \\
\hline Delayed cued recall - single & $14 \cdot 1$ & $4 \cdot 8$ & $16 \cdot 1^{*}$ & $5 \cdot 2$ & $5 \cdot 2$ & $4 \cdot 6$ & 4.9 & 3.9 \\
\hline Delayed cued recall - dual & 11.9 & $5 \cdot 7$ & $12 \cdot 8^{*}$ & $5 \cdot 9$ & $2 \cdot 8$ & $3 \cdot 1$ & $2 \cdot 4$ & $3 \cdot 6$ \\
\hline Delayed free recall - concrete & $9 \cdot 4$ & $5 \cdot 5$ & $9 \cdot 8$ & $6 \cdot 6$ & 1.5 & $1 \cdot 7$ & $3 \cdot 2^{*}$ & $2 \cdot 4$ \\
\hline Delayed free recall - abstract & $4 \cdot 6$ & $3 \cdot 1$ & $5 \cdot 7$ & 3.5 & 0.7 & $1 \cdot 2$ & 0.7 & 0.8 \\
\hline \multicolumn{9}{|l|}{ Semantic memory } \\
\hline Verbal fluency - easy & $19 \cdot 4$ & $4 \cdot 2$ & $19 \cdot 3$ & 4.9 & $17 \cdot 0$ & $6 \cdot 2$ & $16 \cdot 0$ & $6 \cdot 9$ \\
\hline Verbal fluency - hard & $16 \cdot 5$ & $4 \cdot 3$ & $15 \cdot 6$ & $3 \cdot 8$ & $14 \cdot 8$ & $5 \cdot 6$ & $15 \cdot 7$ & $5 \cdot 6$ \\
\hline Category fluency - easy & $16 \cdot 8$ & $3 \cdot 6$ & $16 \cdot 6$ & 3.5 & $14 \cdot 7$ & 4.4 & $12 \cdot 1$ & $5 \cdot 2$ \\
\hline Category fluency - hard & $11 \cdot 1$ & $4 \cdot 0$ & $14 \cdot 4^{*}$ & $5 \cdot 3$ & 11.9 & $2 \cdot 8$ & $10 \cdot 0$ & $4 \cdot 3$ \\
\hline Category verification accuracy - easy & 0.99 & 0.0 & 0.98 & 0.0 & 0.85 & 0.3 & 0.98 & 0.0 \\
\hline Category verification response time - easy & 1044 & 143 & 1035 & 144 & 1223 & 290 & 1252 & 283 \\
\hline Category verification accuracy - hard & 0.90 & $0 \cdot 1$ & 0.89 & 0.1 & 0.81 & 0.3 & 0.89 & 0.1 \\
\hline Category verification response time - hard & 1185 & 143 & 1191 & 163 & 1505 & 353 & 1469 & 286 \\
\hline
\end{tabular}

Mean values were significantly different from those of the control (saccharin) group: ${ }^{*} P<0.05$. † For details of procedures, see p. 415. 
with the analyses of immediate free recall, an analysis of simple interaction effects using the Bonferroni procedure was carried out. Again, there was a significant two-way interaction between treatment and difficulty for older adults $(P<0.05)$. This interaction demonstrated that glucose facilitated older adults' free recall rates for concrete words only.

\section{Semantic memory}

Verbal fluency was analysed in a 2 (glucose $v$. saccharin) $\times 2$ (easy $v$. hard) $\times 2$ (young $v$. old) ANOVA. This analysis revealed greater retrieval from easy compared with hard categories $(F(1,25) 17 \cdot 15$, MSE $8 \cdot 14, P<0 \cdot 01)$, demonstrating a successful difficulty manipulation. All other main effects and interactions were unreliable.

Category fluency was analysed in a 2 (glucose $v$. saccharin) $\times 2$ (easy $v$. hard) $\times 2$ (young $v$. old) ANOVA. This analysis revealed greater retrieval from easy compared with hard categories $(F(1,25)$ 25.96, MSE 10.47, $P<0.01)$, demonstrating a successful difficulty manipulation. There was also a main effect on age group with younger adults retrieving on average more category exemplars than older adults' categories $(F(1,25) 6 \cdot 28$, MSE $28.53, P<0 \cdot 05)$. Notably, there was an interaction between age group and treatment $(F(1,25) 9 \cdot 91$, MSE $10 \cdot 47, P<0 \cdot 01)$. Analysis of simple main effects using the Bonferroni procedure found a significant treatment effect for younger adults only $(P<0 \cdot 05)$.

Semantic verification accuracy was analysed in a 2 (glucose $v$. saccharin) $\times 2$ (easy $v$. hard) $\times 2$ (young $v$. old) ANOVA. This analysis revealed greater accuracy from easy compared with hard categories $(F(1,25) 31.55$, MSE 0.01, $P<0.01)$, demonstrating a successful difficulty manipulation. No other effects were reliable.

Semantic verification response time was analysed in a 2 (glucose $v$. saccharin) $\times 2$ (easy $v$. hard) $\times 2$ (young $v$. old $)$ ANOVA. This analysis revealed quicker responses from easy compared with hard categories $(F(1,25)$ 94.55, MSE $11277, \quad P<0 \cdot 01)$, demonstrating a successful difficulty manipulation. The main effect of age demonstrated quicker responses for younger adults overall $(F(1,25)$ 9.93, MSE $167314, P<0 \cdot 01)$. The difficulty by age group interaction was also significant $(F(1,25) 6 \cdot 03$, MSE $11277, P<0.05)$, demonstrating a greater increase in response time after the difficulty manipulation for older adults.

\section{Physiological measures of mental effort and arousal throughout the testing session}

Heart rate and GSR were collapsed across each task type and difficulty level to increase the reliability of the data.

Heart rate was analysed in a 2 (glucose $v$. saccharin) $\times 2$ (easy $v$. hard) $\times 2$ (semantic $v$. episodic) $\times 2$ (young $v$. old) ANCOVA with baseline heart rate used as a covariate. This analysis found a main effect of age $(F(1,23) 5 \cdot 5$, MSE $85 \cdot 8$, $P<0.05$ ), demonstrating a lower overall heart rate for older adults (73.7 and 70.8 for younger and older adults, respectively). There was also a treatment by age group effect $(F(1,23) 4 \cdot 6$, MSE 56.2, $P<0 \cdot 05)$, demonstrating an increase in heart rate after glucose for older adults only (young: 73.7 and 73.7 for saccharin and glucose, respectively; old: 68.6 and 72.9 for saccharin and glucose, respectively).
GSR was analysed in a 2 (glucose $v$. saccharin) $\times 2$ (easy $v$. hard) $\times 2$ (semantic $v$. episodic) $\times 2$ (young $v$. old) ANCOVA with baseline GSR used as a covariate. This analysis found a main effect of task $(F(1,23) 5 \cdot 8$, MSE 2.4, $P<0.05$ ), demonstrating a lower overall GSR for the episodic memory tasks (9.4 and 10.7 for episodic and semantic memory, respectively). There was also a main effect of difficulty $(F(1,23) 6 \cdot 9$, MSE $0 \cdot 1, P<0 \cdot 05)$, demonstrating higher GSR for the more difficult memory tasks $(9.9$ and 10.2 for easy and hard tasks, respectively). There was also a threeway interaction between treatment, difficulty and age group $(F(1,23) 6 \cdot 1$, MSE $0 \cdot 06, P<0 \cdot 05)$. This interaction demonstrated that for younger adults the change in GSR from easy to difficult task was equivalent between treatment $(0 \cdot 39$ and 0.31 for saccharin and glucose, respectively) compared with older adults (0.07 and 0.39 for saccharin and glucose, respectively). So, although there was a general increase in GSR after the difficulty of the task was manipulated, for older adults glucose gave rise to a substantial rise in GSR for the most difficult cognitive tasks.

\section{Discussion}

The current study set out to investigate the circumstances whereby younger and older adults can benefit from moderate increases in blood glucose. We were particularly interested in the distinction between semantic (i.e. the retrieval of overlearnt information) and episodic (the retrieval of item and contextual information) memory retrieval and the effects of increasing task difficulty. While episodic and semantic memory performance has been examined in earlier studies, none of these studies has provided a direct comparison between the two tasks after task difficulty has been manipulated, in both younger and older adults.

The most robust facilitation was found for episodic memory, which adds to the growing body of research suggesting glucose specifically boosts those functions supported by the hippocampal region. This was seen in immediate and delayed recall and for both younger and older adults. Notably, task difficulty did not exaggerate the glucose facilitation effect. However, the important factor might be that the demands on an individual's cognitive capability are stretched sufficiently to elicit a performance improvement. Examples of this can be observed in Table 2. For immediate cued recall under single task conditions there was significant glucose-related facilitation, yet in the dual task condition the effect disappears. Perhaps under these conditions the additional resource provided by a glucose-containing drink is insufficient as the conditions are too difficult. So, an individual's capability may be stretched to a limit where the glucose dose is insufficient to prevent a reduction in the glucose supply to those structures supporting memory processes (e.g. the hippocampus). The present finding would be consistent with the older adults' data, where there are failures to find glucose facilitation even in the easy task conditions. Therefore, to determine the optimal dose it is necessary to consider not only individual differences in glucose regulation efficiency, which are typically observed between younger and older adults, but also the extent to which the difficulty of the task depletes the glucose reserve in the brain. Interestingly, previous research has suggested that the type of difficulty manipulation 
might be crucial (Riby et al. 2004; Meikle et al. 2005). For instance, Sunram-Lea et al. (2002) found that in younger adults a concurrent motor task during an episodic memory task was crucial to demonstrate glucose facilitation. However, Riby et al. (2004) failed to observe greater facilitation of episodic remembering when a secondary card-sorting task was employed.

A central issue in the present research was to investigate whether memory enhancement extends to semantic tasks, particularly under demanding task conditions. Such findings would be consistent with the idea that memory retrieval in general can benefit from moderate increases in blood glucose. Overall, there was little evidence that glucose can boost semantic memory retrieval. This was the case even when the difficulty of the tasks was manipulated. If traditional measures of effort (accuracy and reaction time) are any indication of the demands of the task, in all cases we were successful in our difficulty manipulation. Furthermore, our measure of GSR indicated that the semantic tasks overall were more difficult. However, the only facilitation observed was for the difficult category fluency task for younger adults. In fact, the special status of the hippocampus in episodic memory has been questioned. Manns et al. (2003) provide data that indicate the hippocampus is involved in semantic as well as episodic memory. The critical factor may also be memory retrieval rather than specifically retrieval from episodic memory (see Allen et al. 1996). Riby (2004) argued that the magnitude of the glucose effect could range from memory tasks requiring retrieval of episodic information (i.e. item and contextual), retrieval of semantic information (overlearnt factual/item only) and retrieval of short-term working memories, with boosts in performance being larger the more of these retrieval operations are being engaged. Interestingly, the finding of facilitation only in younger adults on this task might relate to the relative sparing of semantic memory in ageing. In ageing, semantic memory performance remains stable or even improves (Bowles, 1993). Older adults may have had enough cognitive resource to accomplish this task. So, for the younger adults the difficulty of this task was a key factor. No doubt task difficulty and complexity have a role in the glucose effect, but overall the present results are best explained by a domainspecific account. That is, glucose primarily acts on the hippocampal region, which is known to support episodic memory.

The analysis of the physiological data demonstrated that the GSR measurement was sensitive in detecting differences in task difficulty. Previous research has clearly indicated a relationship between mental effort and GSR (e.g. Pechineda \& Smith, 1996). So, we can be fairly confident in our difficulty manipulations in the present investigation. Also, an important concern for any research investigating the impact of a pharmacological substance on cognitive performance is that the effects may be related to unintentional changes in physiological state (Meikle et al. 2004). For older adults there was an increase in GSR and heart rate in response to glucose treatment. In fact, previous research has suggested that aspects of physiological arousal may be involved in the delivery of additional glucose to the brain (Kennedy \& Scholey, 2000). Future work is clearly warranted in order to investigate how arousal interacts with the glucose effect.
As mentioned earlier, previous research has found older adults to be particularly responsive to glucose ingestion. In the present investigation there was no evidence of an age effect. What should be noted is that older adults may have had insufficient capacity to promote performance improvements after the consumption of the $25 \mathrm{~g}$ glucose drink. Indeed, selected studies have observed $50 \mathrm{~g}$ glucose to be the optimal dose, rather than $25 \mathrm{~g}$, in an elderly population (e.g. Manning et al. 1998). A further issue is that blood glucose regulation efficiency declines with age, which might impact on the glucose facilitation effect (Messier \& Gagnon, 1996). It has been found that individuals with a good ability to regulate their glucose levels will benefit more from additional glucose resources to aid performance. In comparison, individuals with poor glucose regulation will be unable to utilize such additional resources efficiently, resulting in either no boost or a negative impact on performance (e.g. Riby et al. 2004; see also Messier et al. 2003 where the opposite pattern was observed). Therefore, our glucose enhancement effects for older adults might be an underestimation of the utility of glucose in this population. Due to an already demanding testing session (i.e. continuous measurement of heart rate and GSR throughout cognitive testing) we chose not to sample blood to investigate this issue but clearly this is an important consideration. Although measurements of blood glucose were not taken, on the basis of our earlier research glucose levels would be expected to be raised after $10 \mathrm{~min}$ and to have achieved their maximum by approximately 25 min (Meikle et al. 2004). Thus, this experiment was conducted in the period when blood glucose levels were rising to their maximum. One possibility, however, is that glucose levels were at a plateau or decreasing for those cognitive tasks completed in the second half of the testing session.

With the specific tasks used in the present study, task domain seems to be the crucial moderator variable rather than task difficulty. Such findings do not exclude the presence of other glucose-sensitive conditions, but tasks with an episodic memory component are particularly responsive to glucose ingestion. Given that the degree of cognitive difficulty has been demonstrated elsewhere to impact on the magnitude of facilitation on memory tasks (e.g. Kennedy \& Scholey, 2000 - working memory; Sunram-Lea et al. 2002 - episodic memory), further work systematically examining the influence of task domain and task difficulty is warranted. Regardless, the results of the present investigation add to the bulk of evidence, which point to the involvement of the hippocampal region in the glucose facilitation effect. Although the precise mechanisms remain to be elucidated, two candidate mechanisms have been proposed. First, an increase in acetylcholine synthesis and release in response to glucose ingestion could promote facilitation (e.g. Messier et al. 1990). Second, since the hippocampus is densely populated with insulin receptors, a rise in insulin after glucose ingestion might promote glucose utilization (Craft et al. 1994). In summary, episodic memory is crucial to everyday functioning and is known to impact on quality of life. So, future work examining the potential to alleviate poor memory functioning by simple dietary interventions such as administering glucose is a worthwhile line of enquiry. Although minimizing deficits in memory by improvements in glucose regulation might be a more useful strategy. 


\section{Acknowledgements}

We would like to thank the Wellcome Trust for supporting this study. The authors would also like to thank Valerie Gunn for her helpful comments.

\section{References}

Allen JB, Gross AM, Aloia MS \& Billingsley C (1996) The effects of glucose on nonmemory cognitive functioning in the elderly. $\mathrm{Neu}$ ropsychologia 34, 459-465.

Bowles NL (1993) Semantic processes that serve picture naming. In Adult Information Processing: Limit on Loss, pp. 303-326 [J Cerella, J Rybash, W Hoyer and M Commons, editors]. London: Academic Press.

Brown WP (1978) Belfast Category Norms, 1971-77. Belfast: Department of Psychology, Queen's University of Belfast.

Coltheart M (1981) The MRC Psycholinguistic Database. $Q J$ Exp Psychol A 33, 497-505.

Craft S, Murphy C \& Wemstrom J (1994) Glucose effects on complex memory and nonmemory tasks - the influence of age, sex, and glucoregulatory response. Psychobiology 22, 2, 95-105.

Craik FIM (1986) A functional account of age differences in memory. In Human Memory and Cognitive Capabilities, Mechanisms, and Performances, pp. 409-422 [F Klix and H Hagendorf, editors]. New York: Elsevier Science.

Dunnett S (1991) Cholinergic grafts, memory and ageing. Trends Neurosci 14, 371-376.

Folstein MF, Folstein SE \& McHugh PR (1975) 'Mini-mental state': a practical method for grading cognition stroke of patients for the clinicians. J Psychiatr Res 12, 189-198.

Foster JK, Lidder PG \& Sunram S (1998) Glucose and memory: fractionation of enhancement effects?" Psychopharmacology 137, $259-270$.

Fucetola R, Newcomer JW, Craft S \& Melson AK (1999) Age and dose dependent glucose induced increases in memory and attention in schizophrenia. Psychiatry Res 88, 1, 1-13.

Hall JL, Gonder-Frederick LA, Chewning WW, Silveira J \& Gold PE (1989) Glucose enhancement of performance on memory tests in young and aged humans. Neuropsychologia 27, 1129-1138.

Kaplan RJ, Greenwood CE, Winocur G \& Wolever TMS (2000) Cognitive performance is associated with glucose regulation in healthy elderly persons and can be enhanced with glucose and dietary carbohydrates. Am J Clin Nutr 72, 825-836.

Kennedy DO \& Scholey AB (2000) Glucose administration, heart rate and cognitive performance: effects of increasing mental effort. Psychopharmacology 149, 63-71.

Kennedy DO \& Scholey AB (2003) Ginseng: potential for the enhancement of cognitive performance and mood. Pharmacol Biochem Behav 75, 687-700.
Lezak MD (1995) Neuropsychological Assessment, 2nd ed. p. 545. New York: Oxford University Press.

Manning CA, Ragozzino ME \& Gold PE (1993) Glucose enhancement of memory in patients with probable senile dementia of the Alzheimer's type. Neurobiol Aging 14, 523-528.

Manning CA, Stone WS, Korol DL \& Gold PE (1998) Glucose enhancement of 24-h memory retrieval in healthy elderly humans. Behav Brain Res 93, 1/2, 71-76.

Manns JR, Hopkins RO \& Squire LR (2003) Semantic memory and the human hippocampus. Neuron 38, 127-133.

Meikle A, Riby LM \& Stollery B (2004) The impact of glucose ingestion and gluco-regulatory control on cognitive performance: a comparison of younger and older adults. Hum Psychopharmacol: Clinical and Experimental 19, 523-535.

Meikle A, Riby LM \& Stollery B (2005) Memory processing and the glucose facilitation effect: the effects of stimulus difficulty and memory load. Nutr Neurosci (In the Press).

Messier C (2004) Glucose improvement of memory: a review. Eur J Pharmacol 490, 33-57.

Messier C, Durkin T, Mrabet O \& Destrade C (1990) Memoryimproving action of glucose - indirect evidence for a facilitation of hippocampal acetylcholine synthesis. Behav Brain Res 39, 2, $135-143$.

Messier C \& Gagnon M (1996) Glucose regulation and cognitive functions: relation to Alzheimer's disease and diabetes. Behav Brain Res 75, 1/2, 1-11.

Messier C, Gagnon M \& Knott V (1997) Effect of glucose and peripheral glucose regulation on memory in the elderly. Neurobiol Aging 18, 297-304.

Messier C, Tsiakas M, Gagnon M, Desrochers A \& Awad N (2003) Effect of age and glucoregulation on cognitive performance. Neurobiol Aging 24, 985-1003.

Pechineda A \& Smith CA (1996) The affective significance of skin conductance activity during a difficult problem solving task. Cogn Emotion 10, 481-503.

Riby LM (2004) The impact of age and task domain on cognitive performance: a meta-analytic review of the glucose facilitation effect. Brain Impairment 5, 2, 145-165.

Riby LM, Meikle A \& Glover C (2004) The effects of age, glucose ingestion and gluco-regulatory control on episodic memory. Age Ageing 33, 483-487.

Saller CF \& Kreamer LD (1991) Glucose-concentrations in brain and blood - regulation by dopamine receptor subtypes. Brain Res $\mathbf{5 4 6}$, 235-240.

Scholey AB, Harper S \& Kennedy DO (2001) Cognitive demand and blood glucose. Physiol Behav 73, 585-592.

Sunram-Lea SI, Foster JK, Durlach P \& Perez C (2002) Investigation into the significance of task difficulty and divided allocation of resources on the glucose memory facilitation effect. Psychopharmacology 160, 387-397. 